\title{
Origin of surface layer on common substrates for functional material films probed by ellipsometry
}

\author{
A.I. Belyaeva, A.A. Galuza, A.D. Kudlenko \\ National Technical University "Kharkov Politechnical Institute", 21 Frunze st., 61002 Kharkov, Ukraine \\ E-mail: belyayeva@kpi.kharkov.ua
}

\begin{abstract}
A multiple angle ellipsometric method is used to investigate thin film layers on common substrates (gadolinium gallium garnet-GGG, sapphire- $\mathrm{Al}_{2} \mathrm{O}_{3}$, and glass ceramic sitall) for functional material films. The method evaluates fundamental optical constants and thicknesses of the layers. Dielectric functions for the surface layers on such kind of plates have been determined. Coincidence up to the third decimal point in refractive index value $\left(n_{f}\right)$ was shown. Errors for the thickness of surface layer $\left(d_{f}\right)$ is not more than $3 \%$. It is shown that the dielectric properties of microscopically rough layers of thickness $\sim 10-45 \mathrm{~nm}$ can be accurately modeled in the homogeneous thin layer approximation. The thickness and origin of the surface layer on substrates are found out. Experimental data analysis allows to make some conclusions concerning the origin of this layer. It is a specific amorphous damaged layer that remains after taking out stressed surface layer via chemical polishing. The corrosion processes on glass ceramic seems to be very similar to those occurring on GGG and sapphire single crystals.
\end{abstract}

Keywords: ellipsometry, damaged layer, interface layer, optical constants.

Paper received 04.09.02; accepted for publication 18.03.03.

\section{Introduction}

The paper is devoted to investigation of surface layers on common substrates for various functional material films.

Optical properties of a substance on the surface are always somehow different from ones of the bulk material. It happens due to peculiarities of technology, surface processing, aging and may be connected with oxidation, random mechanical damages, roughness of the surface, and with combination of this factors. Ellipsometric technique is sensitive to these changes and allows to develop various physical models of this layer to amend understanding the origin of the surface layer.

To create a film with optimal parameters one should have proper information about roughness of the substrate surface and about possible interface layer between the film and substrate. Besides, one should understand how these peculiarities effect properties of the system in general.

Since optical methods (e.g., ellipsometry) are widely used to investigate optical properties of film materials for opto- and microelectronics, it is necessary to have complete information about optical constants of the substrates. Nevertheless, there is very little information of this kind in literature. Knowledge of the origin of sur- face layers that directly contact the functional material film is necessary to estimate properties of film - substrate whole system in general.

We chose three kind of materials for the investigation. They are: single-crystal gadolinium gallium garnet $(\mathrm{GdGaG})$, and sapphire $\left(\mathrm{Al}_{2} \mathrm{O}_{3}\right)$, and amorphous glass ceramic - sitall. These materials are currently popular as substrates for various functional films.

$\mathrm{GdGaG}$ is a basic substrate material for rear earth ferrogarnet epitaxial films. There is no information about the surface layer on $\mathrm{GdGaG}$ substrate now, though its influence on epitaxial film properties is obvious and may result, for instance, in the epitaxial film being in nonpreferrable orientation. So, during manufacturing of $\mathrm{GdGaG}$ substrates an interface layer forms between air and the material. This layer can significantly change optical properties of the whole system.

Since discovery of HTSC many materials were tested as substrates for $\mathrm{Y}-\mathrm{Ba}-\mathrm{Cu}-\mathrm{O}$ films. Analysis of literature showed that sapphire is one of the most popular materials for this purpose now, since sapphire substrates are available in large sizes at low cost [1]. Information about the substrate, in particular about the surface layer, is fairly important since in many measurements (e.g. optical and r.f.) properties of the substrate may obscure those 


\section{A.I. Belyaeva et al.: Origin of surface layer on common substrates for ...}

of the film. It is obvious that if the damaged layer was not removed, then crystallographic orientation of substrate would not important under epitaxy. Specifically, sometimes just damaged layer on sapphire substrate prevented the growing film to get proper orientation. The X-ray data of depositions on sapphire substrates showed that the growth had no preferable orientation [2]. The X-ray analysis showed that as-deposited films were amorphous [3]. To obtain the desired orientation of $\mathrm{Y}-\mathrm{Ba}-\mathrm{Cu}-\mathrm{O}$ films $c$-axis, researches covered the substrate with buffer $\mathrm{MgO}$ layer.

One of the major problems of the modern optical industry is creating low noise optical devices for infrared spectral range. The mirrors cooled down to cryogenic temperature are the main part of such devices. Cooling allows to reduce noise significantly and amplify the signal. However, the mirrors should not change their shape after cooling that may result in mistrusting of the device. In the case of deeply cooled optical devices the input signal intensity can be compared with the noise intensity that is why mirrors in such systems must be perfect. The traditional materials, such as glass, can not with stand such temperature gradients, as take place during cryogenic cooling. That is why the cryogenic mirrors are usually made of the so-called non-traditional materials such as glass ceramic (called sitall as well), melted quartz, beryllium, and so on [4]. Though metals can not compete with silicate materials in terms of quality and reflecting properties, and in terms of isotropy and stability.

Among the non-traditional materials sitall CO-115M (glass ceramic $\mathrm{Li}_{2} \mathrm{O}-\mathrm{Al}_{2} \mathrm{O}_{3}-\mathrm{P}_{2} \mathrm{O}_{5}-\mathrm{SiO}_{2}$ ) is one of the most promising, since it has good optical and mechanical properties and can be easily polished [4]. We chose this a very material for our investigation. From data available, one can expect that corrosion processes connected with surface processing glass ceramic are close to ones of sapphire, though there is no such information in literature.

We chose the ellipsometry as the main experimental technique, since it does not spoil the sample and is very sensible even to negligible layers. Besides, it allows to build models of the damage surface layer. We used also electron microscopy investigations to determine the model more exactly. The ellipsometry and electron microscopy are based on different physical mechanisms and complement each other in the study of roughness.

All the samples were selectively chemically etched using etchant that remove only stressed phase formed as a result of a mechanical treatment.

\section{Experimental techniques and specimens}

The optical characteristics of the sample surfaces were investigated by ellipsometry at fixed wavelength (632.8 $\mathrm{nm}$ ) and modeled as a homogeneous thin layer on a substrate [5]. The "thickness" of this apparent layer and its optical indices were obtained from the ellipsometric data.

The ellipsometric measurements were taken at different angles of incidence at room temperature with null-
LEF-3M-1 laser ellipsometer $(\lambda=632.8 \mathrm{~nm})$ described in details in [6]. The incidence angles were varied from $50^{\circ}$ up to $70^{\circ}$. Four-zone averaging was used to reduce errors to about $\leq \pm 0.01^{\circ}$ for $\Psi$ and $\leq \pm 0.05^{\circ}$ for $\Delta$ in measuring ellipsometric characteristics. These results were used to determine optical constants of the sample and to model its surface layer.

In case of anisotropic samples, it is necessary to make some additional investigations. So, we developed a technique for determining the difference of refractive indexes of ordinary and extraordinary rays. Schematic of the optical set-up is given in Fig. 1.

Here the sample is placed between crossed polarizers. Changes of light polarization state, while passing through this system or completely linearly polarized light decomposition on the ordinary and extraordinary rays in birefringent crystal are shown in Fig. 2.

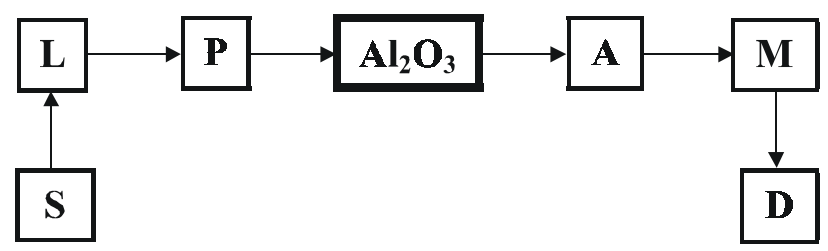

Fig. 1. Schematic of the optical set-up. $\mathrm{Al}_{2} \mathrm{O}_{3}-$ sample under study, $\mathrm{S}$ - light source, $\mathrm{L}$ - lens, $\mathrm{P}$ and $\mathrm{A}$ - polarizer and analyzer, $\mathrm{M}$ - monochromator, D - detector.

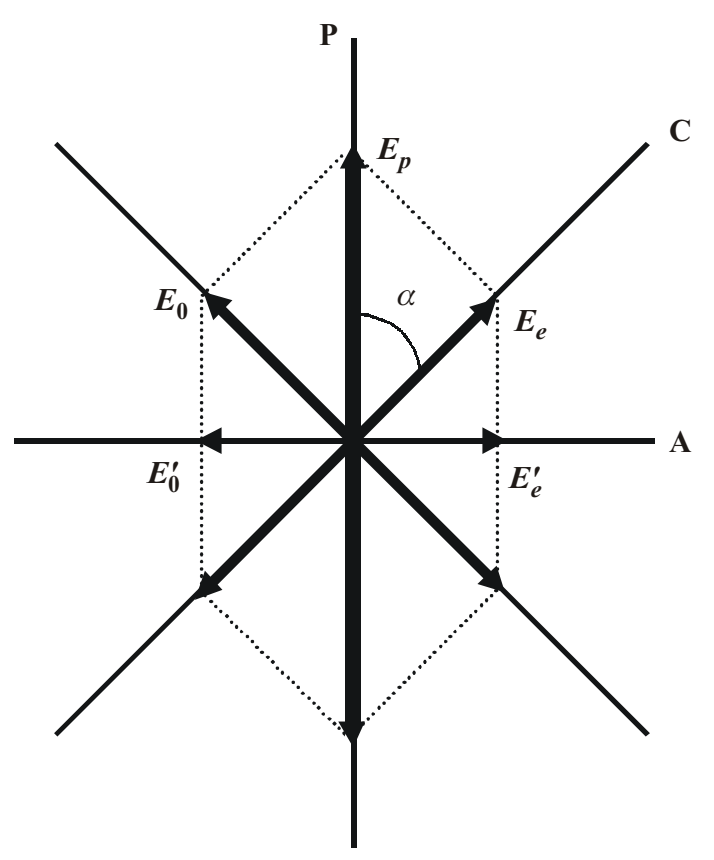

Fig. 2. Completely linearly polarized light decomposition on the ordinary and extraordinary rays in birefringent crystal. $\mathrm{P}$ polyarizer axis, $\mathrm{C}$-optical axis of crystal; $\alpha=45^{\circ} . E_{0}$ and $E_{e}-$ electrical vector for ordinary and extraordinary rays, correspondingly.

SQO, 6(1), 2003 


\section{A.I. Belyaeva et al.: Origin of surface layer on common substrates for ...}

Two light waves, which have mutually orthogonal polarization, get difference in phase $\delta$ after living plate with thickness $h$ due to the difference of refractive indexes $n_{0}$ and $n_{e}$ :

$\delta=\frac{2 \pi}{\lambda}\left(n_{o}-n_{e}\right) h$

where $\lambda$ - light wavelength.

The wave components, which are A-axis projections $E_{0}$ and $E_{e}$, or $E_{0}$ and $E_{e}$, will transmit through the analyzer A. These oscillations have different phase $\delta$ and interfere with each other. Light intensity after A for every spectral components is:

$I_{\perp}=E_{p}^{2} \sin ^{2}(\delta / 2)$,

where $\perp$ for crossed $\mathrm{P}$ и $\mathrm{A}, E_{p}$ - completely linearly polarized light amplitude after P. We see from (2) that A keeps out the light $\left(\lambda_{m}\right)$ under condition that:

$\delta=2 \pi m, m=0,1,2 \ldots$
For difference in path $\Delta=\left(n_{0}-n_{e}\right) h$ (3) will be:

$\Delta=\left(n_{0}-n_{e}\right) h=m \lambda_{m}$.

Thus, interference minimums will be for $\lambda_{m}$, when difference in phase is even numbers of $\pi$ and difference in path is equal to even numbers of $\lambda$.

We carried out experiment for observing interference of polarized rays, which was obtained as a result of birefringence in the sapphire single-crystal. Within visible spectrum there are seven interference minimums (Fig. 3). We determine wavelength for all them and using equations (1)-(3) estimated the refraction indexes difference of ordinary and extraordinary rays for the sapphire plate under study. The thickness of the plate was $800 \mathrm{~mm}$ and $\Delta n$ turned out 0.009 .

\section{Experimental results}

The Figs 4-6 show experimental dependencies (shown with points) of ellipsometric parameters $\Psi$ and $\Delta$ versus

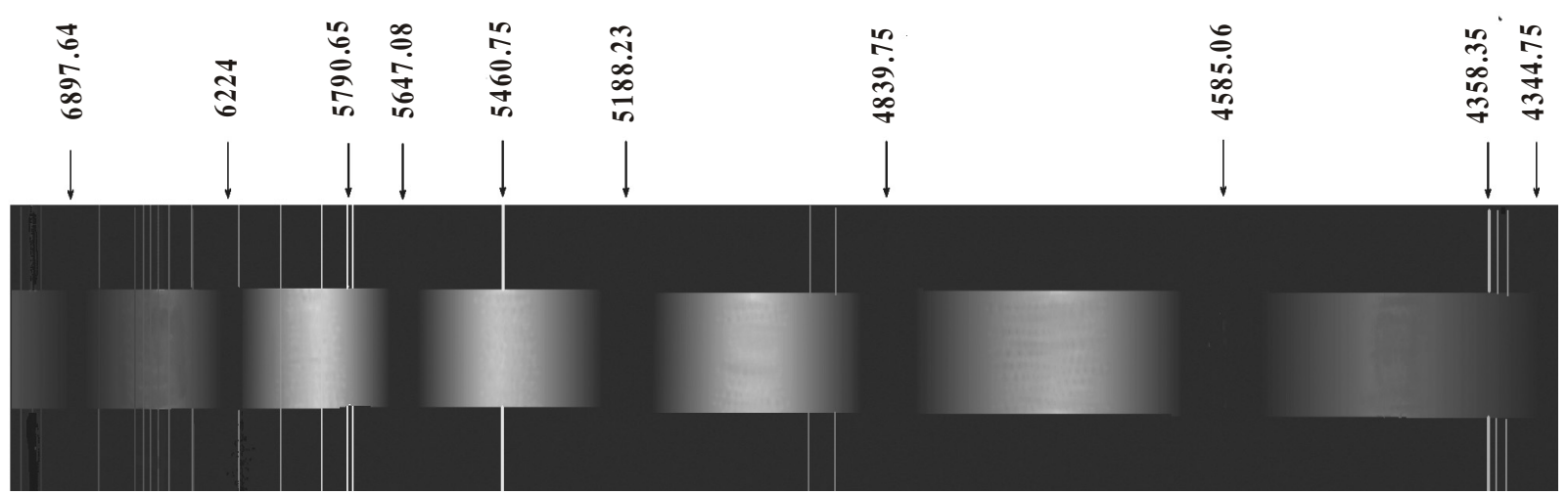

Fig. 3. Interference bands of sapphire single-crystal.

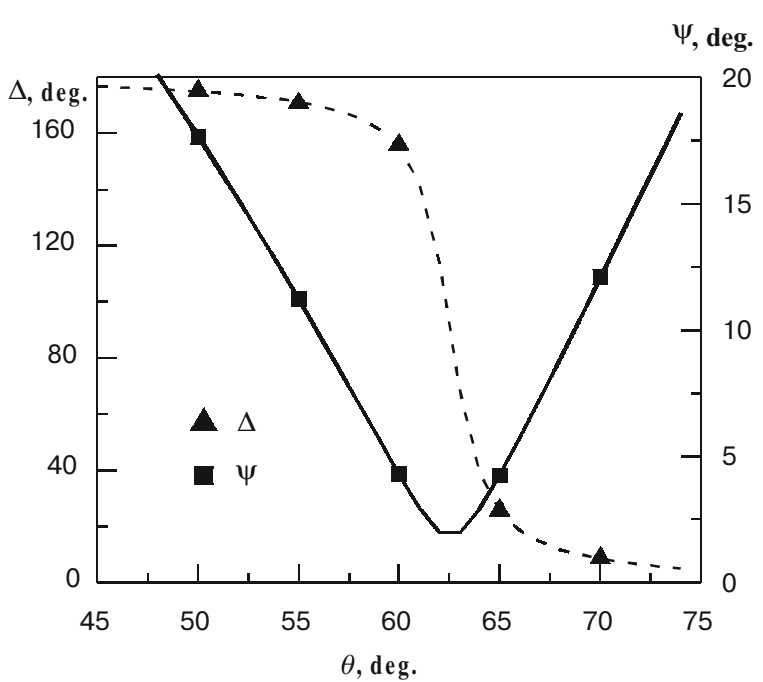

Fig. 4. Dependence of ellipsometric parameters $\Psi$ and $\Delta$ versus incidence angle (curves - calculation in model film-GGG substrate with experimental points). Experimental error is in the experimental point size.

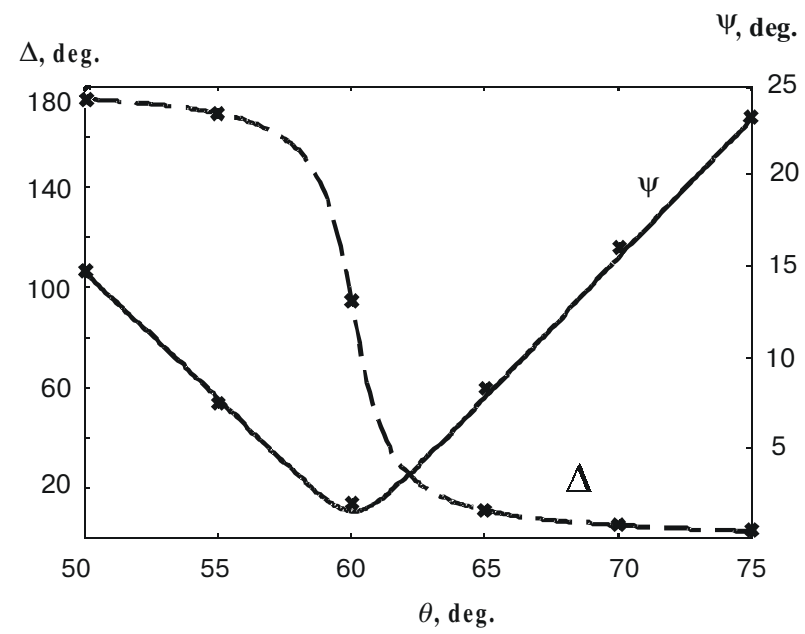

Fig. 5. Dependence of ellipsometric parameters $\Psi$ and $\Delta$ versus incidence angle (curves - calculation within model film-sapphire substrate with experimental points). Experimental error is in the experimental point size. 
A.I. Belyaeva et al.: Origin of surface layer on common substrates for ...

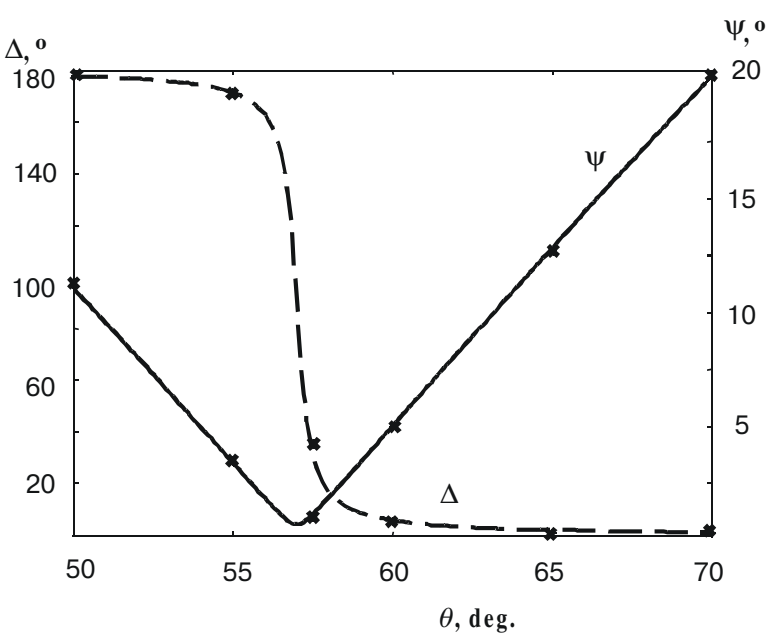

Fig. 6. Dependence of ellipsometric parameters $\Psi$ and $\Delta$ versus incidence angle (curves - calculation within model film-sitall substrate with experimental points). Experimental error is in the experimental point size.

incidence angle for GGG (Fig. 4), sapphire (Fig. 5) and sitall (Fig. 6). Measurements were carried out within angle range from $50^{\circ}$ up to $80^{\circ}$. Native GGG growth surfaces of $\{211\}$ type were chemically etched with phosphoric acid at temperature $t=280{ }^{\circ} \mathrm{C}$ according to technique similar to the one described in [7].

The ellipsometric experimental data analysis shows that the surfaces of all investigated samples (GGG, sapphire, and sitall) are not native, but have surface layer (film). But experimental values $\Psi_{\text {exp }}$ and $\Delta_{\text {exp }}$ for all samples are not far from thus for bulk material. It means that $n_{s}$ and $n_{f}$ (and Bruster angles) are near-values.

We investigated our samples by light scattering technique to reveal macroroughness. But there was not any scattering. So, ellipsometric angles variations were not connected with the surface macroroughness.

But we revealed microroughness by means of electron microscopy analysis films of replica covered by $\mathrm{Pt}$. The surface of the samples under study was irregular with chance structure and peculiarities at about $20-30 \mathrm{~nm}$ distance. Last value correlates with the experimental data for thickness of the surface layer. Thus, the model of homogeneous film for microrough surface of the substrate is very good.

\section{Analysis of errors}

Table 1 shows influence of real experimental errors on modeling results for single layer structure air-film-GGG substrate for GGG $\left(n_{s}=1.98, n_{f}=1.87, d_{f}=33.2 \mathrm{~nm}\right.$, $\left.\theta=65^{\circ}, \lambda=632.8 \mathrm{~nm}\right)$. Experimental errors equal to $\pm 0.01^{\circ}$ for $\Psi$ and $\pm 0.05^{\circ}$ for $\Delta$. One can see that $n_{f}=1.87$ is determined precisely up to the third decimal digit and $d_{f}$ error does not exceed more then $3 \%$.
Table 1. Influence of real experimental errors on modeling results for single layer structure air-film-GGG substrate for GGG $\left(n_{s}=1.98, n_{f}=1.87, d_{f}=33.2 \mathrm{~nm}, \theta=65^{\circ}, \lambda=632.8 \mathrm{~nm}\right)$.

\begin{tabular}{llll}
\hline \hline$\Delta_{\text {exp }}{ }^{\circ}$ & $\Psi_{\text {exp }}{ }^{\circ}$ & $n_{f}$ & $d_{f}, \mathrm{~nm}$ \\
\hline $25.792+0.00$ & $4.237+0.00$ & 1.8682 & 32.4330 \\
\hline $25.792+0.10$ & $4.237+0.05$ & 1.8681 & 33.1463 \\
\hline $25.792-0.10$ & $4.237-0.05$ & 1.8681 & 31.6889 \\
\hline $25.792-0.10$ & $4.237+0.05$ & 1.8695 & 33.4486 \\
\hline $25.792+0.10$ & $4.237-0.05$ & 1.8666 & 31.3885 \\
\hline \hline
\end{tabular}

\section{Damage layer model and origin}

Ellipsometric investigations of $\mathrm{GdGaG}$, sapphire, and sitall surfaces (Figs 4-6) were used to prove presence of interface layer and to develop its physical model. Single homogeneous surface film model and effective medium model describing microroughness of the surface were considered. The surface was analyzed within the physical model of a film with refractive index $n_{f}$ and thickness $d_{f}$ on homogeneous substrate with refractive index $n_{s}$, thickness of the substrate assumed infinite. This system (film substrate) is placed in homogeneous isotropic media (air) with known refractive index $\left(n_{a}\right)$. It is supposed that substrate, film, and media are transparent $\left(k_{a}=k_{f}=k_{s}=\right.$ $=0)$. We investigated the case of homogeneous along thickness film $\left(n_{f}=\operatorname{const}\left(d_{f}\right)\right)$.

These results were used to estimate physical model and calculate optical constants of surface layers. We used mean square deviation as the criterion of a model being adequate. The sum of squared errors

$\Phi(\mathbf{x})=\frac{1}{N} \sqrt{\sum_{i=1}^{N}\left(\Psi_{\text {calc }}^{i}(\mathbf{x})-\Psi_{\text {exp }}^{i}\right)^{2}+\left(\Delta_{\text {calc }}^{i}(\mathbf{x})-\Delta_{\text {exp }}^{i}\right)^{2}}$

is minimized leading to the set of optical parameters characterizing the surface. $N$ - is the number of data points, subscripts exp and calc refer to the experimental and calculated parameters.

Modeling of the real sample was performed within single layer model. Namely, ambient (air)-film-substrate. The surface layer (roughness) on the top of an infinite layer of bulk substrate material was modeled as a homogeneous film with apparent optical constant $n_{s}, n_{f}$ and thickness $d_{f}$. A schematic of this geometry is shown in Fig. 7 a,b,c for all ofthe three samples. Fitting the results for two models proved that single layer model gives much better approximation than the clean surface one. The (3) is $1.2^{\circ}$ for the two-phase model (sample-air) and $0.1^{\circ}$ for our air-film-substrate model. So, taking surface layer into account improved the quality of the approximation for each sample about 5-50 times comparing with clean surface model.

It is shown that in all three case (GGG, sapphire, and glass ceramic substrates) there is a damaged surface layer (with refractive index a bit smaller then the one of 


\section{A.I. Belyaeva et al.: Origin of surface layer on common substrates for ...}

\begin{tabular}{ll}
$\operatorname{Air}\left(n_{a}=1\right)$ & $\boldsymbol{a}$ \\
\hline Film $\left(n_{f}=1.87, d_{f}=33 \mathrm{~nm}\right)$ \\
\hline $\begin{array}{l}\text { Substrate: GdGaG } \\
\left(n_{s}=1.98 \pm 0.001\right)\end{array}$ \\
\hline
\end{tabular}

\begin{tabular}{ll}
$\operatorname{Air}\left(n_{a}=1\right)$ & $\boldsymbol{b}$ \\
\hline $\operatorname{Film}\left(n_{f}=1.68, d_{f}=32 \mathrm{~nm}\right)$ \\
\hline
\end{tabular}

Substrate $\mathbf{A l}_{\mathbf{2}} \mathbf{O}_{3}$

$\left(n_{s}=1.769 \pm 0.001\right)$ \begin{tabular}{ll}
$\operatorname{Air}\left(n_{a}=1\right)$ & $c$ \\
\hline Film $\left(n_{f}=1.53, d_{f}=45 \mathrm{~nm}\right)$ & \\
\hline
\end{tabular}

Substrate: sitall

( $\left.n_{s}=1.55 \pm 0.001\right)$

Fig. 7. Models of GGG (a), sapphire (b), and glass ceramic (c) substrates

the bulk material), which is the result of mechanical processing (polishing) of the sample and can not be removed by chemical polishing. Such layer may be formed on both single-crystal substrate (such as GGG and sapphire) and ceramic.

The analysis of experimental results allows to make some conclusions concerning the origin of this layer. Thus, the fact that refractive indexes of the layer and bulk material are close brings about the conclusion that this layer is amorphous damaged layer, which is the result of mechanical processing and remains after removing stressed layer via chemical polishing. The fact that damage layer lasts after chemical polishing make it possible to rule out its connection with stress under mechanical polishing and offer the other mechanism of its formation. Under mechanical polishing of the surface the damage amorphous layer may be formed near the surface that is connected with melting of the surface layer and its rapid cooling. The cooling rate is more than the crystallization one, which leads to deregulation of surface layer structure.

Refractive index difference for crystalline and amorphous material is very small, it is not more than $0.1(\Delta n=$ $\left.=n_{c}-n_{a} \approx 0.1\right)$ [8]. This value coincides with our values for all our materials (GGG, sapphire, and ceramic) in the frame of offered models. But this value is more than enough for so sensitive technique as ellipsometry, which allows to identify such kind of layer.

Presence of this layer should be taken into account when using such plates as substrates for growing various epitaxial films since the damaged layer may result in interface layer between the film and substrate that would distort properties of the system in general.

Difficulties, which are well known from literature, arising while growing epitaxial films on GGG and HTSC films on sapphire are connected with not taking into account the damaged surface layer. The fact that the growing films turned out to be amorphous confirms that the found layer is amorphous as well.

\section{Conclusions}

1. $\mathrm{GdGaG}, \mathrm{Al}_{2} \mathrm{O}_{3}$, and glass ceramic (sitall) substrates were investigated using ellipsometry technique.

2. A layer on the surface of $\mathrm{GdGaG}$, sapphire, and glass ceramic was found. Refractive index $n_{f}$ and thickness $d_{f}$ of this layer were determined. It was shown that all three samples have significant surface damaged layer of about $30-45 \mathrm{~nm}$ thick.

SQO, 6(1), 2003
3. Complex investigation using ellipsometric method and method of polarized rays interference allowed to determine both refractive index and birefringence (difference of refractive indices for ordinary and extraordinary rays) of a sapphire single-crystal.

4. It was shown that in the all three cases studied (GdGaG, sapphire, and glass ceramic) interface layer in a system substrate-interface layer-air is a damaged surface layer (with refractive index smaller then the one of the bulk sample), that is the result of mechanical polishing the samples. Besides, it is proved that taking this layer into account improved quality of modeling by 5 to 50 times comparing with the clean surface model.

5. Experimental data analysis allows to make some conclusions concerning the origin of this layer. It is a specific amorphous damaged layer that remains after etching out stressed surface layer via chemical polishing.

6. Such layer may be formed on both single-crystal (GdGaG and sapphire) and glass ceramic substrates as a result of mechanical processing. One should take this layer into account if using such plates as substrates for growing various epitaxial films, since it can be a source of an interface layer between substrate and film and distort properties of the whole system.

\section{References}

1. A.B. Beresin, C.W. Yuan, A.L. Lozanne. Y-Ba-Cu-O thin films grown on Sapphire with epitaxial $\mathrm{MgO}$ buffer layers // Appl. Phys. Lett. 57 (1), p. 90-92 (1990).

2. R.M. Silver, A.B. Beresin, M. Wendman, A.L.de Lozanne. As-deposited superconducting $\mathrm{Y}-\mathrm{Ba}-\mathrm{Cu}-\mathrm{O}$ thin films on $\mathrm{Si}$, $\mathrm{Al}_{2} \mathrm{O}_{3}$ and $\mathrm{SrTiO}_{3}$ substrates // Appl. Phys. Lett. 52 (25), p. 2174-2176 (1988).

3. R.M. Silver, J. Talvacchio, A.L. de Lozanne. Sputter deposition of Y-Ba-Cu-O thin films // Appl. Phys. Lett. 51 (25), p. 2149-2151 (1987).

4. A.I. Belyaeva, T.G. Grebennik, S.A. Pogorelova et al. Cooling system of cryogenic mirrors made of non-trivial materials: The preprint of the Institute for Low Temperature Physics \& Engineering AS Ukraine, Kharkov, 1990, N10, 33 p.

5. A.I. Belyaeva, A.A. Galuza, T.G. Grebennik, V.P. Yuriev. Determination of the parameters of a transparent film deposited onto an absorbing substrate by the ellipsometry method // Optics and spectroscopy 87 (6), p. 952-955 (1999).

6. A.I. Belyaeva, A.A. Galuza, T.G. Grebennik, V.P. Yuriev. Determination of the Refractive Index and Thickness of a Transparent Film and Double Layer Systems on any Substrate via Cryogenic Spectrometric Ellipsometry // Advances in Cryogenic Engineering (Materials) 46 A, p.435-442 (2000).

7. J. Bastorfield. The chemical polishing of Yttrium iron garnet // J. Phys. D. Ser.2, 2, p. 1159-1161 (1969).

8. Handbook of Optical Constants of Solids, Vol.1, Ed. E.A. Palik, Academic Press, Orlando, FL, (1985). 